\title{
Ka Band System and Propagation Effects on System Performance
}

\author{
R. Acosta and S. Johnson \\ R.Acosta@GRC.NASA.gov \\ Sandra.Johnson@GRC.NASA.gov \\ Phone: 216-433-6640 Fax: 216-433-6371 \\ NASA Glenn Research Center \\ Cleveland, Ohio 44135
}

\begin{abstract}
The Advanced Communication Technology Satellite (ACTS) is an experimental communications satellite system launched in September 1993. ACTS introduces many new technologies, including operation in the Ka frequency band. ACTS uses multiple hopping narrow beams and very small aperture terminal (VSAT) technology to establish a system availability of $99.5 \%$ for a bit-error-rates of $5 \times 10^{-7}$ or better over the continental United States. In order to maintain a minimum system availability of $99.5 \%$ over all US rain zones ACTS uses an adaptive rain fade compensation protocol to reduce the impact of signal attenuation resulting from propagation effects. The purpose of this paper is to present the results of system and sub-system characterization considering the statistical effects of system variances due to thermal and propagation effects over 6 years of ACTS operation including inclined orbit.
\end{abstract}

\subsection{Introduction}

The primary objective of the technology verification experiment (TVE) program is to obtain a deeper understanding and to fully statistically characterize Ka band sub-systems over the life of ACTS. [1]. Beginning in August of 1998, ACTS began to operate in an inclined orbit of 0.8 degrees per year.

The objectives are being achieved by making long-term measurements (120 sites years) at multiple sites and analyzing the collected data. The technology experiments have been concentrated but not limited to, four specific areas. In Section 2, the T1 (1.54 Mbps) VSAT System Availability Experiment is described. This experiment consists of developing a cumulative distribution function analysis of propagation effects and system effects (e.g., multibeam antenna pointing error due to thermal distortions). Section 3 covers the Statistical Rain Fade Compensation Characterization Experiment, where the statistical impact of adding $10 \mathrm{~dB}$ of coding gain to a $\mathrm{Ka}$ band link is established. The Statistical Characterization of Ka Band Propagation Effects on the Communication Link Performance Experiment is described in Section 4. In this experiment, the propagation effects (e.g., antenna wetting and link attenuation) in a $\mathrm{Ka}$ band link are evaluated using cumulative distribution function analysis with seven measurements sites using ACTS propagation terminals (APT) as well as with several Ultra Small Aperture Terminals (USATs). Section 5 discusses the Multibeam Antenna Performance Experiment, in which the multibeam antenna pointing accuracy is statistically evaluated and thermal distortion effects are characterized.

At the conclusion of the ACTS technology experiment program, upper bound levels for all of the impairments to the satellite signal loss caused by various propagation phenomena will be known. In addition, the collected data will provide a lasting database of $30 / 20 \mathrm{GHz}$ propagation and system data for communications satellite builders.

\subsection{T1 VSAT System Availability Experiment}

The T1 VSAT system availability experiments analyze the effects of Ka band system variances and propagation on the ACTS T1 VSAT performance using statistical performance indexes such as system fade availability over several years [2]. Table I summarizes the site parameters that were considered in the experiment. All seven sites listed were used for the pre-inclined orbit phase of operation, and three sites (Boulder, CO; Clarksburg, MD; and Boca Raton, FL) remain for the inclined orbit phase. 


\begin{tabular}{|c|c|c|c|c|c|c|}
\hline $\begin{array}{c}\text { Site } \\
\text { Location }\end{array}$ & $\begin{array}{c}\text { \# Days } \\
\text { Data } \\
\text { Collected }\end{array}$ & $\begin{array}{c}\text { Latitude } \\
\text { (Degs.) }\end{array}$ & $\begin{array}{c}\text { Longitude } \\
\text { (Degs.) }\end{array}$ & Elevation & $\begin{array}{c}\text { Elevation } \\
\text { Angle } \\
\text { (Degs.) }\end{array}$ & $\begin{array}{c}\text { CCIR Rain } \\
\text { Zone }\end{array}$ \\
\hline Pasadena, CA & 98.5289 & $34.201 \mathrm{~N}$ & $118.174 \mathrm{~W}$ & $346.15 \mathrm{~m}$ & 45.749 & $\mathrm{E}$ \\
\hline Reston, VA & 306.3360 & $38.950 \mathrm{~N}$ & $77.346 \mathrm{~W}$ & $113 \mathrm{~m}$ & 39.14 & $\mathrm{~K}$ \\
\hline Clarksburg, MD & 331.1631 & $39.220 \mathrm{~N}$ & $77.280 \mathrm{~W}$ & $184 \mathrm{~m}$ & 38.86 & $\mathrm{~K}$ \\
\hline Boulder, CO & 407.1492 & $39.993 \mathrm{~N}$ & $105.253 \mathrm{~W}$ & $1638 \mathrm{~m}$ & 43.416 & $\mathrm{E}$ \\
\hline Boca Raton, FL & 150.4268 & $26.373 \mathrm{~N}$ & $74.066 \mathrm{~W}$ & $24 \mathrm{~m}$ & 52.193 & $\mathrm{~N}$ \\
\hline Bogota, Columbia & 131.8965 & $4.629 \mathrm{~S}$ & $80.102 \mathrm{~W}$ & $2630 \mathrm{~m}$ & 59.257 & $\mathrm{~N}$ \\
\hline Quito, Ecuador & 75.1742 & $0.207 \mathrm{~S}$ & $78.493 \mathrm{~W}$ & $2956 \mathrm{~m}$ & 64.806 & $\mathrm{~N}$ \\
\hline
\end{tabular}

TABLE I - Summary of VSATs Site Parameters

The ACTS T1 VSAT has the following features: 12 watts transmit power, antenna size of $1.2 \mathrm{~m}$ or $2.4 \mathrm{~m}$, operating frequencies of $29.236 \mathrm{GHz}$ and $29.291 \mathrm{GHz}$ (uplink) and 19.44 $\mathrm{GHz}$ (downlink), Serial Minimum Shift Keying (SMSK) modulation, adaptive fade compensation using burst rate reduction and convolutional coding, and a throughput of 1.792 Mbps. In addition, the VSAT does not include a beacon receiver - the downlink signal to noise value is estimated from the reference energy burst.

The system fade availability experiment goal included determining the overall statistical availability of a number of T1 VSATs in different rain zones. In the analysis, all outage types were categorized and summarized including rain at the VSAT as well as rain at the control station.

\subsection{Summary of Results}

The total percentage of time the VSAT was available to the user (Total Availability), and the percentage of time the outages were only due to rain at the VSAT (VSAT RFA) and at the VSAT plus the control station (System RFA) are shown in Figures $\mathbf{l a}$ and $\mathbf{1 b}$. The total

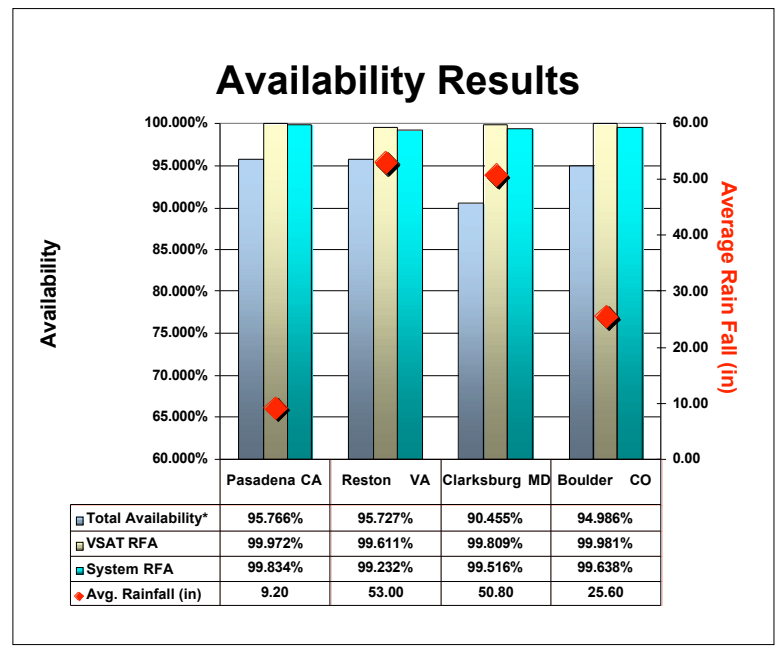

Figure 1a - Summary of Results

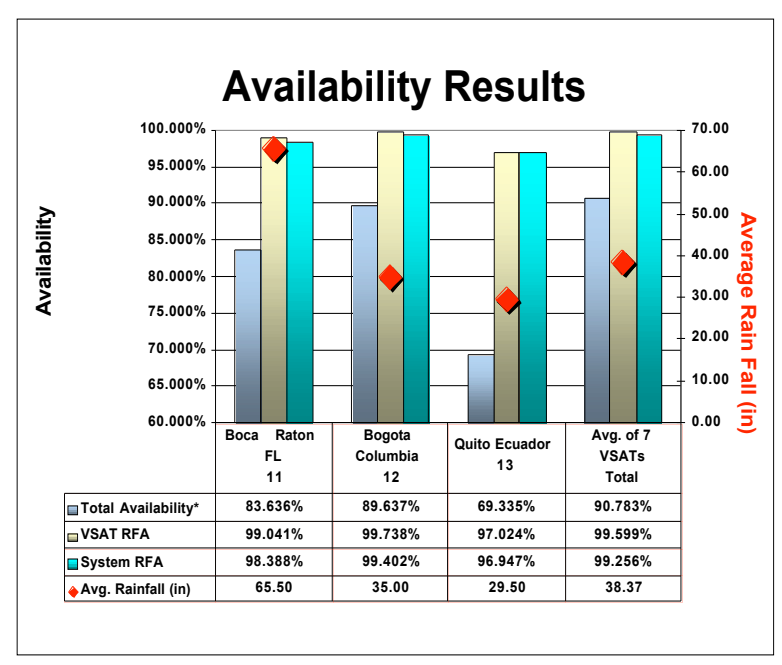

Figure $1 \mathrm{~b}$ - Summary of Results

average annual rainfall in the location is shown for comparison. The worst case VSAT rain fade availability occurred at the VSAT located in Quito, Ecuador. Its availability over the 75 hours of operation was $97 \%$. Compensation was not enabled for this particular VSAT during the experiment period. Although the largest rainfall amounts are not in this location, the type of rain experienced in this location is more likely to cause outages.

Although rain outages are a major contributor to the overall availability of the VSAT, other outage types contribute significantly to the downtime that a user might experience. Because ACTS is an experimental system, extended downtimes were expected as hardware and software anomalies were addressed. Hardware failures, which include time for diagnosis of the problem, time for an operational part to be shipped to the site and installed, and time for 
testing to assure operation, contributed to approximately $57 \%$ of the time that a VSAT was non-operational. Modifications were made to the VSAT and to operational procedures to decrease this downtime. These modifications included increased remote monitoring, diagnosis and reset capabilities; additional spare units; additional training of on-site users; and hardier cables and connectors.

\subsection{Rain Fade Compensation Characterization Experiment}

The ACTS Adaptive Rain Fade Compensation Protocol is a process whereby a VSAT data channel BER performance is automatically enhanced during a period of signal attenuation due to rain or thermal distortion on the spacecraft antenna $[3,4]$. The rain fade compensation protocol provides $10 \mathrm{~dB}$ of margin by reducing burst rates by half and invoking rate convolutional code, constraint length 5 . The result is a reduction of the $110 \mathrm{Mbps}$ burst rates to $55 \mathrm{Msps}$ and the $27.5 \mathrm{Mbps}$ burst rates to $13.75 \mathrm{Msps}$. The protocol is adaptive in that it includes a decision process so that fade compensation is implemented only when needed. The decision process, which determines the need for compensation in real time, makes use of the downlink signal level, a FADED threshold and a CLEAR threshold. The downlink signal level estimation is made by each VSAT and transmitted to the NASA ground station. Hardware and software in the VSAT samples the demodulator "eye" pattern of the center bit of 1's and 0's triplets in the downlink burst 9600 times in a $75 \mathrm{~ms}$ superframe. The Mean to Variance method is then applied to the samples resulting in an estimated value of the signal to noise level once every $75 \mathrm{~ms}$. Each estimated value is stored in a first in first out register. The signal level reported to the NGS is the average of 13 estimated consecutive values, i.e., it is a 1 second moving average that is reported once every $150 \mathrm{msec}$. An experiment system diagram is presented in Figure 2.

In this experiment configuration, two T1 VSATs are located next to each other at the NASA Glenn Research Center in Cleveland, Ohio. One of the T1 VSATs (\#7) is configured so that adaptive fade compensation is enabled. The other T1 VSAT (\#11) has adaptive fade compensation disabled, which precludes the compensation technique from ever being enacted.

The ACTS adaptive rain fade compensation protocol was designed to ensure a T1 VSAT bit error rate of no worse than $5 \times 10^{-7}$ for at least $99.5 \%$ of the time. The T1 VSATs are designed to operate with 5 and $3 \mathrm{~dB}$ uplink and downlink margin, respectively. The implementation of FADED or CLEAR operation is designed to accommodate a fade rate of at least $0.25 \mathrm{~dB}$ per second, to have no impact on VSAT throughput, and to be seamless in the transition from CLEAR to FADED operation and back.

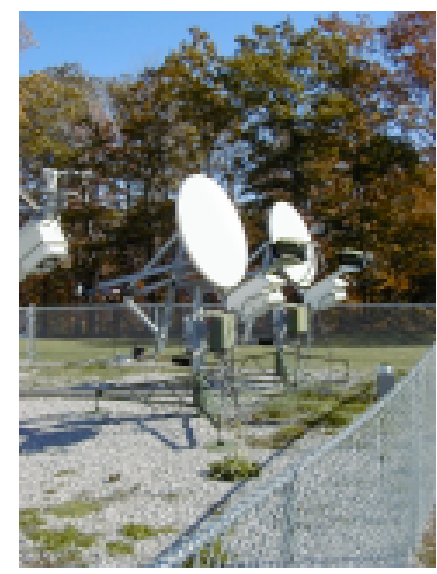

Figure 2 Rain Fade Compensation Experiment System

\subsection{Summary of Results}

The cumulative distribution function for the measured uplink bit error rate (BER), the measured downlink BER, and the fade cumulative distribution function for both the VSAT with compensation enabled (VSAT \#7) and the VSAT with compensation disabled (VSAT \#11) is shown in Figure 3. 
The $10 \mathrm{~dB}$ above clear sky margin translated to an increase of $0.5 \%$ (at $1 \times 10^{-6} \mathrm{BER}$ ) system availability in a medium rain zone during the five-month experimental period.

\section{TVE 918 - Rain Events - Cumulative CDF}

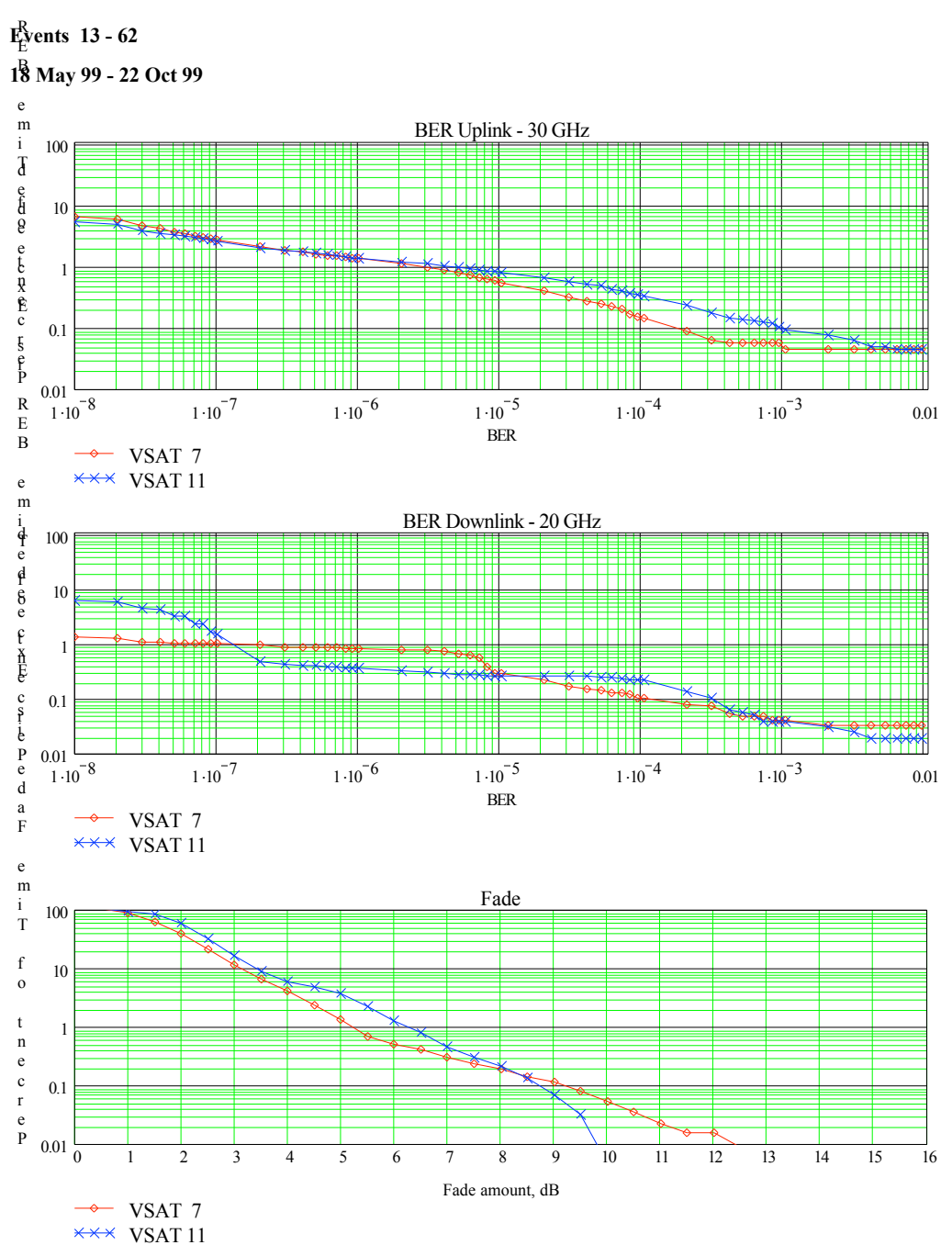

Figure 3. BER and Fade Cumulative Distribution Functions

The protocol was considered more than adequate for all rain zones in CONUS. System availability was found to exceed $99.5 \%$ for all rain zones tested with ACTS using the fade compensation system on demand. System fades were found to be between 3-5 dB, while rain fade exceeded this range for about $5 \%$ of time. For all other regions the extra $10 \mathrm{~dB}$ was an overdesign margin since few ( $1 \%$ of time at best) rain events with fades greater than $10 \mathrm{~dB}$ were encountered.

\subsection{Ka Band Propagation Effects on the Communication Link Performance Experiment}

Rain attenuation is the dominant propagation impairment at Ka-band frequencies. Rain attenuation is a function of frequency, elevation angle, polarization angle, rain intensity, rain drop size distribution and raindrop temperature. The communication link performance experiment included more than seven sites in North America and lasted for a period of five years [5]. Propagation factors that affect $\mathrm{Ka}$ band satellite links operating at moderate to high elevation angles include: gaseous absorption, cloud attenuation, melting layer attenuation, rain attenuation and rain/ice depolarization. 
Gaseous absorption, cloud attenuation, melting layer attenuation, and rain attenuation are absorptive effects producing both signal attenuation and a proportionate increase in thermal noise received at the antenna port. Systems employing orthogonal polarization to implement frequency reuse suffer from interference produced by rain and ice depolarization. Tropospheric scintillation is non-absorptive and produces signal attenuation as well as enhancements. From the ACTS beacon data collected it is seen that the fade ratio $(30 \mathrm{GHz} /$ $20 \mathrm{GHz}$ ) from light rain, which is mainly composed of smaller drops, tends to be large compared to heavy rain, which tends to have a higher proportion of larger drops.

\subsection{Propagation Experiment Description and Results}

The ACTS beacon data has shown that fade depth at $27.5 \mathrm{GHz}$ under moderate elevation angles exceeds $20 \mathrm{~dB}$ for $0.1 \%$ of a typical year. Fading becomes worse for low-elevations and higher rainfall climates. Fade rates associated with rain attenuation are found to be distributed in a log-normal fashion with a median fade rate around $0.1 \mathrm{~dB} / \mathrm{sec}$. Fade rate increases with the depth of attenuation and can reach values as large as $1 \mathrm{~dB} / \mathrm{sec}$. Little difference exists between the fade rates on the increasing and decreasing side of an attenuation event.

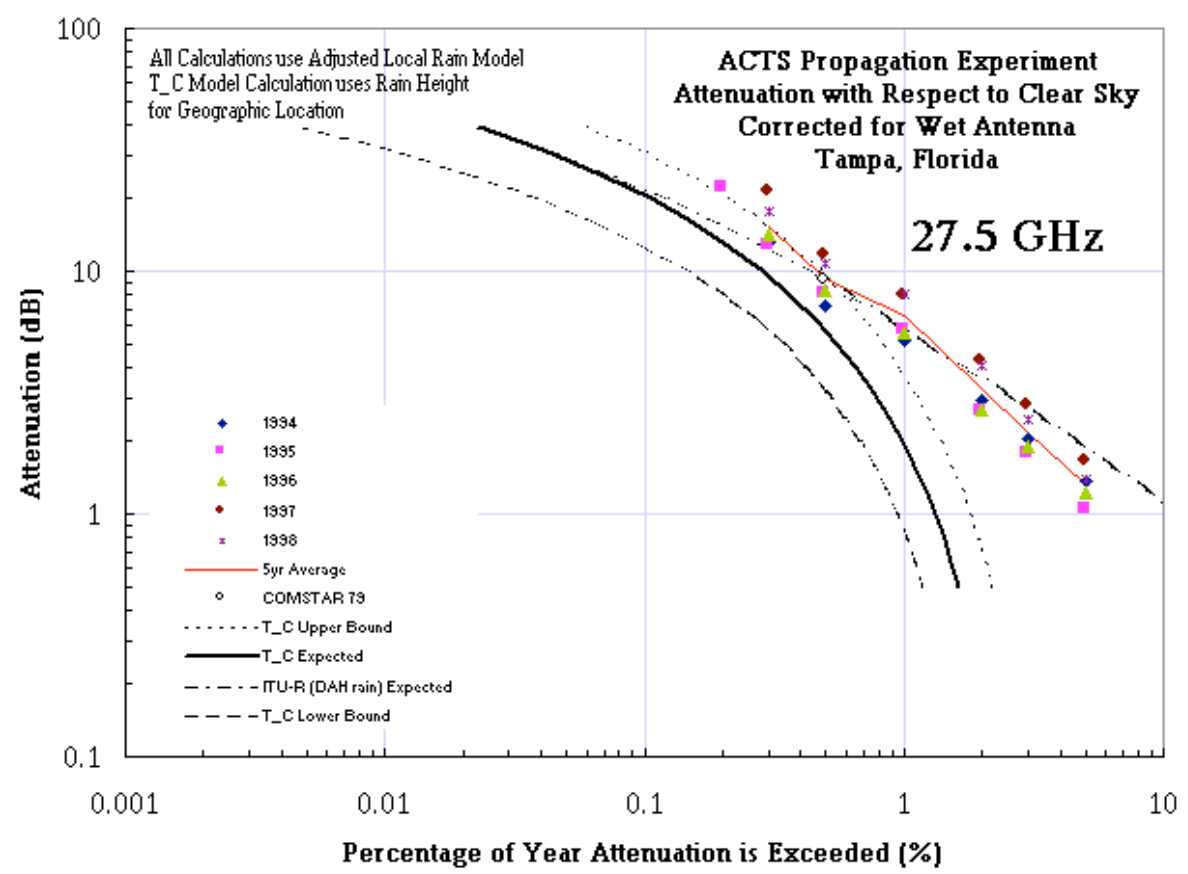

A five years cumulative distribution function of typical observations made at 20.2 and $27.5 \mathrm{GHz}$ by one the seven ACTS propagation sites (Tampa, Florida) is presented in Figure 4. Figure 5 shows the scintillation statistics observed at the Florida location. The data was collected using the beacon signals on the ACTS satellite, with an elevation angle of 51 degrees. Total path attenuation, which includes gaseous absorption and other clear-air effects, are included in the distributions. Also shown in Figure 4 is the rain rate distribution. It is seen that annual raining time for the measurement site is around $5 \%$. 


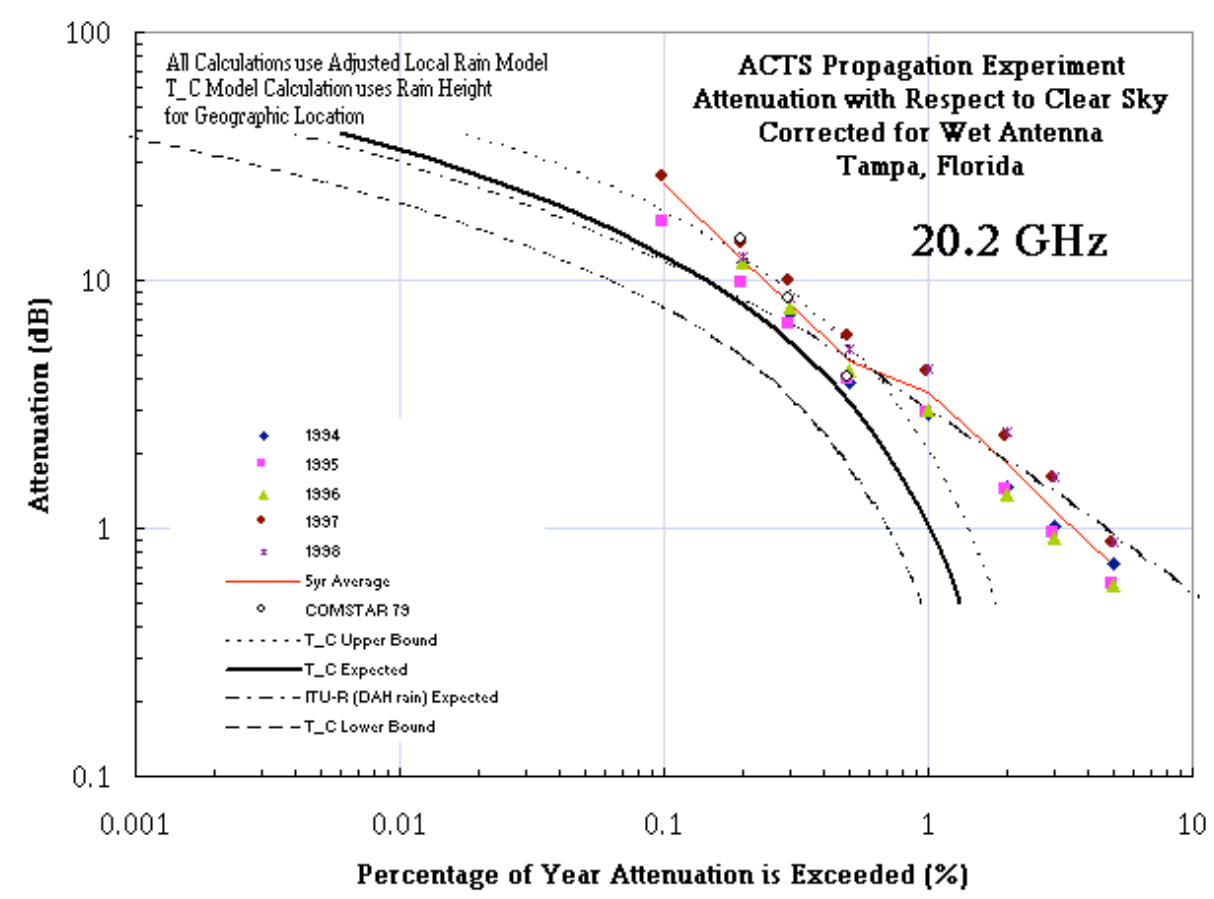

Five Year CDF in Tampa, Florida

Figure 4.

Annual time percentage for which rain attenuation is present along the observation is somewhat higher due to fact that rain attenuation is produced by the presence of rain along the satellite and the rain rate distribution pertains only to a point measurement near the earth station antenna. Due to the presence of other factors such as cloud attenuation, raining time along the path cannot be easily discerned. As shown in the figure, fade depth at 27.5 $\mathrm{GHz}$ under moderate elevation angles exceed $20 \mathrm{~dB}$ for $0.1 \%$ of an average year. Fading becomes worse for low-elevation and /or severe rainfall climates.

Figure 5 ranks each applicable rain attenuation model in the order in which they compare with the actual measured cumulative distribution functions.

\begin{tabular}{|c|c|c|c|c|}
\hline Model & $\begin{array}{c}\text { ITU } \\
\text { Overall }\end{array}$ & $\begin{array}{c}\text { ITU } \\
15-35 \mathrm{GHz}\end{array}$ & $\begin{array}{l}\text { ACTS } \\
20 \mathrm{GHz}\end{array}$ & $\begin{array}{l}\text { ACTS } \\
27 \mathrm{GHz}\end{array}$ \\
\hline$D A H$ & 1 & 2 & 1 & 1 \\
\hline$I T U$ & 2 & 3 & 3 & 4 \\
\hline ExCell & 3 & & 2 & 2 \\
\hline CCIR & & & 5 & 5 \\
\hline $\begin{array}{c}\text { Two } \\
\text { Comp }\end{array}$ & & & 4 & 3 \\
\hline
\end{tabular}

Figure 5. Ranking of Accuracy of Prediction Models vs. Measured Rain Attenuation 


\subsection{Wet Antenna}

This section describes an experimental treatment of the contribution of wet reflector antennas to the signal path losses in a Ka band. The amount of water in ground antenna systems can cause additional signal degradation from the expected propagation attenuation due to rain in the satellite path [6]. In Ka band communication systems, an additional margin should be included to account for the wetness of the antenna system. The objective of the antenna wetting experiment consisted of measuring the magnitude of the effect of water on the feed and reflector on the total signal attenuation and its correlation with rain rates measured with a tipping bucket system. The experiment consisted of deploying and operating two identical receive only VSAT reflector ground systems located side-by-side at an elevation angle of 51 degrees. The dry reflector was protected from the rain expected on the aperture plane. The received $\mathrm{CW} 20.2 \mathrm{GHz} \mathrm{RF}$ signal was digitally recorded at _ second sampling rate and filtered with a $40 \mathrm{KHz}$ band pass filter centered at $70 \mathrm{MHz}$. The receiver had a dynamic range of about $30 \mathrm{~dB}$, therefore only fades up to $30 \mathrm{~dB}$ can be resolved with the system. In addition to measuring the received signal strength, a small weather station was operated next to the VSAT terminals. Rainfall data was collected using a tipping bucket rain gauge. The experiment location was selected to include tropical and sub-tropical rain characteristics and was located in Cocoa, Florida. The data collection period extended between October to December 1997 and February to August 1, 1999.

In general these results showed that feed wetness is the main contributor to the system losses, with reflector wetness being a lesser factor. The water in the feed aperture distorts the electric field's distribution of the feed creating a high perturbation on the feed standing wave ratio (SWR). The reflector losses can be explained by additional scattering losses due to raindrop's size at the surface of the reflector. This creates a distorted reflector surface that reduces the antenna gain by several $\mathrm{dBs}$ in the worst case.

\subsubsection{Statistical Experiment Antenna Wetting Results}

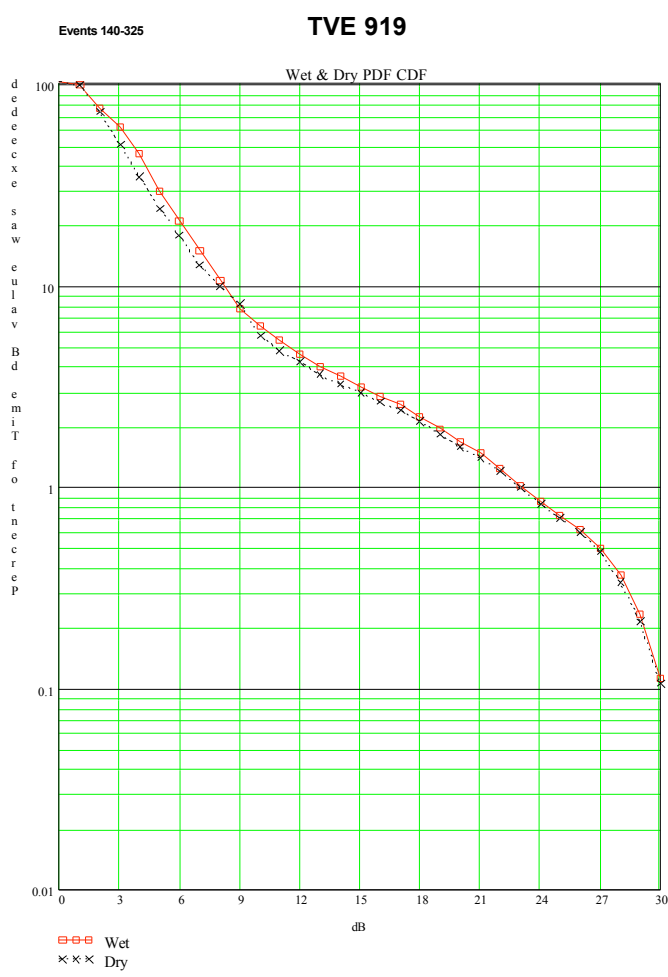

Figure 6. Cumulative Distribution for the Dry Wetting Effects

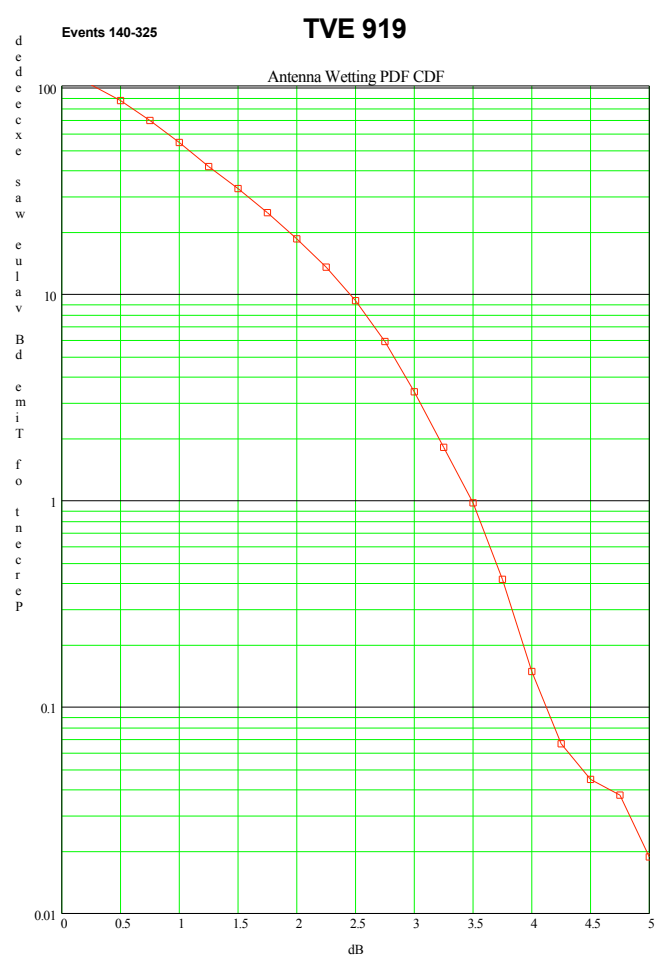

Figure 7. Combined Antenna and Wet Antenna

A total of 2000 experiment hours (rain and dry weather) were collected. 300 hours were rain events. Figures 6 and 7 shows the cumulative distribution for the dry antenna and wet antenna. There is good correlation between antenna wetting and rain rates $(>60 \%)$. One observation which can be drawn from these results is that the cumulative fade distribution 
measured using a wet antenna will be several $\mathrm{dB}$ worse than the fade statistics measured with a dry antenna.

\subsection{Multibeam Antenna Performance Experiment}

The ACTS system introduces several new technologies including a multibeam antenna (MBA) system operating at $\mathrm{Ka}$ band [7]. The antenna produces 3 fixed and 47 rapidly reconfigurable spot beams with approximately 0.3 degrees beamwidth and gains of approximately $50 \mathrm{dBi}$. One the most significant aspects of the satellite communication system design is the accurate estimation of antenna performance degradation. The thermal or dynamic distortions of a reflector antenna system can have the following effects on far-field antenna power away from the desired locations: 1- Reduced antenna isotropic radiated power; 2- Mispointed main lobe antenna pattern causing the RF power to spread over larger area than desired; 3 Increased antenna pattern sidelobes increasing the potential of interference among adjacent beams of a multiple beam antenna system or with antenna beams of other satellites. A number of MBA performance evaluations have been conducted since the ACTS launch. These evaluations were designed to assess the MBA performance (e.g. beam pointing stability, beam shape, gain, etc.) in the space environment.

\subsection{Experiment Results}

The multibeam antenna's on-orbit test measurements over a six-year period found the MBA performance to be well within the expected range. The transmit and receive beam optimization procedures were successfully executed. In general, on-orbit MBA measurements have shown good performance, but there have been some problems caused by thermal and mechanical disturbances that can easily be corrected in future designs of similarly complex antennas.

On orbit measurements found the ACTS multibeam reflector thermal distortions to be periodic. This periodicity makes it possible to predict and compensate the effects of the rapidly varying thermal events on system performance as a function of the time of day and time of year. Figure 8 shows the temperature distribution function observed at the transmit main reflector and sub-reflector antenna for the five year. Figure 9 shows the temperature distribution function observed at the receive main reflector and sub-reflector antenna for the five years. Notice that there is no detectable degradation of change between minimum and maximum temperature during the life of ACTS.

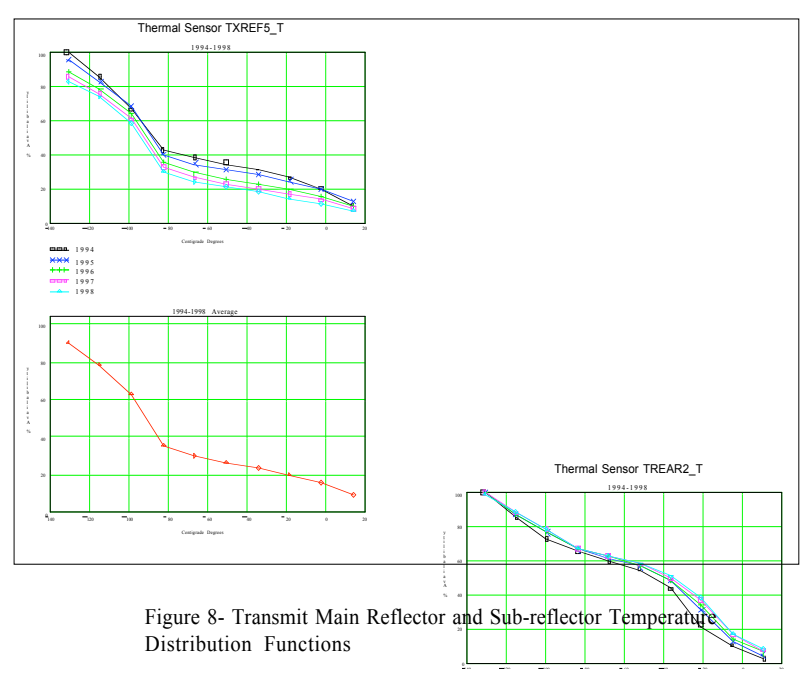

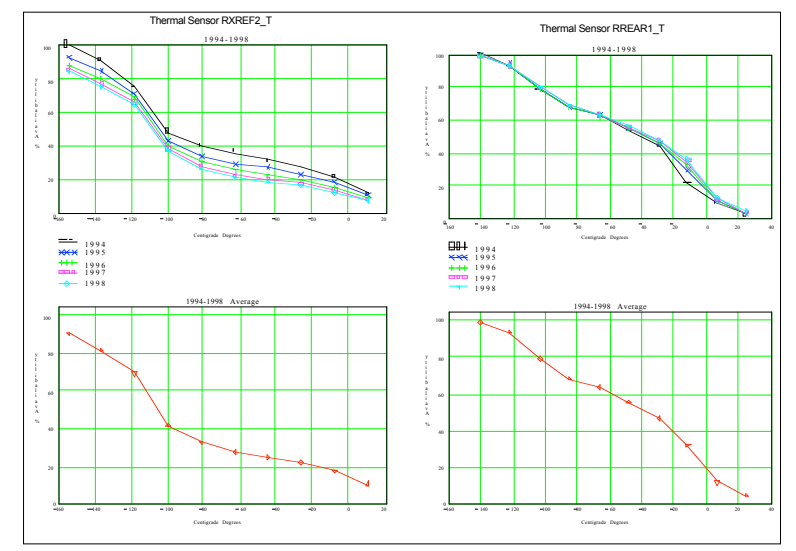

Figure 9- Receive Main Reflector and Sub-reflector Temperature Distribution Functions

In order to compensate for thermal effects, several techniques were designed and tested to improve performance. The compensation of thermal effects makes the west beams (back sub-reflector beams) stable $100 \%$ of the time and the east beams (front sub-reflector) stable about $75 \%$ of the time. Table II describes the system impact with and without compensation for ground stations within the $3 \mathrm{~dB}$ contour. 
Great caution should be exercised in the design of future communications satellites with multibeam reflector systems at $\mathrm{Ka}$ band or higher frequencies using gridded reflector structures (cause of the rapidly varying thermals events) and material like Aztroquartz (with a high coefficient of thermal expansion).

The mechanical oscillations $(1 \mathrm{~Hz}$ vibrations) on the ACTS MBA are very difficult to compensate in flight. Future communications satellites should consider the mechanical design. Specifically its mechanical resonant frequency should be much larger than the maximum mechanically induced frequency by the spacecraft. In the ACTS design, it was known that the resonant frequency was low, but weight constraints prevented the additional structural components from being added.

The yaw control system can affect the very narrow ACTS beam pointing drastically, especially for those ground stations located away from either the sub satellite longitude or the autotrack boresight. Future spacecraft systems should make yaw estimation a primary concern in their design goals. The data collected on the ACTS system yaw estimator show that the yaw error is very sensitive to environmental changes and spacecraft mechanical noises, thus making the yaw estimation process during times outside the sun window inaccurate.

\begin{tabular}{|c|c|c|c|c|c|}
\hline $\begin{array}{c}\text { Type } \\
\text { of Distortion } \\
\text { (Duration) }\end{array}$ & $\begin{array}{c}\text { Beam } \\
\text { Polarization }\end{array}$ & $\begin{array}{c}\text { Magnitude } \\
\text { of Effect }\end{array}$ & $\begin{array}{c}\text { Axis } \\
\text { of Distortion }\end{array}$ & $\begin{array}{c}\text { System } \\
\text { Impact }\end{array}$ & $\begin{array}{c}\text { Compensation } \\
\text { Technique }\end{array}$ \\
\hline $\begin{array}{c}\text { Rapid Varying } \\
(<1 \mathrm{Hr})\end{array}$ & East $(\mathrm{H})$ & $<0.1^{\circ} \circ$ & Roll & $\begin{array}{l}\text { Short Term Impact } \\
\text { Attenuation }<3 \mathrm{~dB}\end{array}$ & $\begin{array}{c}\text { Earth Sensor } \\
\text { Control }\end{array}$ \\
\hline $\begin{array}{c}\text { Diurnal Variation } \\
(<12 \mathrm{Hr})\end{array}$ & $\begin{array}{l}\text { East }(\mathrm{H}) \\
\text { West }(\mathrm{V})\end{array}$ & $<0.2^{\circ}$ & Pitch & $\begin{array}{l}\text { Significant Signal } \\
\text { Attenuation }<6 \mathrm{~dB}\end{array}$ & $\begin{array}{c}\text { Biax Drive } \\
\text { Compensation }\end{array}$ \\
\hline $\begin{array}{c}\text { Quasistatic } \\
(<14 \text { Days })\end{array}$ & $\begin{array}{l}\text { East }(\mathrm{H}) \\
\text { West }(\mathrm{V})\end{array}$ & $\begin{array}{c}<0.8 \circ \\
<0.4 \circ\end{array}$ & $\begin{array}{c}\text { Pitch } \\
\text { Roll }\end{array}$ & $\begin{array}{l}\text { Significant Signal } \\
\text { Attenuation }<8 \mathrm{~dB}\end{array}$ & $\begin{array}{c}\text { Autotrack } \\
\text { Compensation }\end{array}$ \\
\hline $\begin{array}{c}\text { Vibration } \\
(1 \mathrm{~Hz})\end{array}$ & $\begin{array}{l}\text { East }(\mathrm{H}) \\
\text { West }(\mathrm{V})\end{array}$ & $<0.4 \circ$ & Pitch & Attenuation $<.1 \mathrm{~dB}$ & None \\
\hline
\end{tabular}

TABLE II - System Impact of Thermal Distortions

\subsection{Concluding Remarks}

The ACTS satellite system has been instrumental in adding knowledge of rain fade phenomena so that system designers can better predict the type of availability that can be provided in different rain regions, or how to tailor the system to provide quality availability at low cost. The research conducted using ACTS on rain fade compensation to date has proved that that coding gain and power control can be quite effective in combating rain fading. The Glenn Research Center Space Communication Office-ACTS is currently conducting an aggressive program to investigate all aspects of rain fade compensation including low cost implementation for future Ka-band systems. 


\subsection{References}

[1] R. Acosta, R. Bauer, R. Krawczyk, R. Reinhart, M. Zernic and F. Gargione, "Advanced Communications Technology Satellite (ACTS): Four-Years System Performance", IEEE Journal on Selected Areas in Communications. VOL. 17, No. 2, February 1999.

[2] S. Johnson, "Lessons learned from ACTS T1 VSAT experiment program", $4^{\text {th }} \mathrm{Ka}$ Band Utilization Conference, Venice, Italy 1998.

[3] C. Cox and T. Coney, "ACTS Fade Compensation Protocol Impact on VSAT Bit Error Rate Performance", IEEE Selected Areas in Communications, Vol 12, Number 2 February 1999.

[4] C. Cox and T. Coney, "ACTS Adaptive Rain Fade Compensation Protocol Performance", $4^{\text {th }}$ Ka Band Utilization Conference, Venice, Italy 1998.

[5] R. Bauer, "Ka-Band Propagation Measurements: An Opportunity with the Advanced Communication Technology Satellite (ACTS)", Proceedings of IEEE, Vol. 85, No. 6, June 1997.

[6] R. Acosta," Wet Antenna Effects on Ka Band Low Margin Sysytems", $4^{\text {th }}$ Ka Band Utilization Conference, Venice, Italy 1998.

[7] R. Acosta,"ACTS Multibeam Antenna Analysis and on-orbit performance," $2^{\text {nd }} \mathrm{Ka}$ Band Utilization Conference, Florence, Italy 1996. 